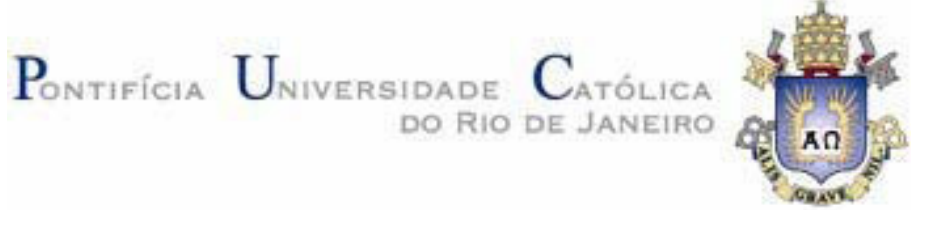

Gustavo Nunes Wagner

\title{
Visualização Interativa de Modelos Massivos de Engenharia na Indústria de Petróleo com o Algoritmo de Voxels Distantes
}

Dissertação de Mestrado

Dissertação apresentada como requisito parcial para obtenção do título de Mestre pelo Programa de PósGraduação em Informática da PUC-Rio.

Orientador: Prof. Marcelo Gattass Co-Orientador: Prof. Alberto Raposo 
Gustavo Nunes Wagner

\section{Visualização Interativa de Modelos Massivos de Engenharia na Indústria de Petróleo com o Algoritmo de Voxels Distantes}

Dissertação apresentada como requisito parcial para obtenção do título de Mestre pelo Programa de PósGraduação em Informática da PUC-Rio. Aprovada pela Comissão Examinadora abaixo assinada.

Prof. Marcelo Gattass

Orientador

Departamento de Informática - PUC-Rio

Prof. Alberto Raposo

Co-Orientador

Departamento de Informática - PUC-Rio

Prof. Marcelo Dreux

Departamento de Informática - PUC-Rio

Dr. Luciano Reis

CENPES - Petrobras

Prof. Waldemar Celes

Departamento de Informática - PUC-Rio

Prof. José Eugenio Leal Coordenador Setorial do Centro Técnico Científico - PUC-Rio

Rio de Janeiro, 9 de abril de 2007 
Todos os direitos reservados. É proibida a reprodução total ou parcial do trabalho sem autorização da universidade, do autor e do orientador.

\section{Gustavo Nunes Wagner}

Engenheiro de Computação graduado pela Pontifícia Universidade Católica do Rio de Janeiro em dezembro de 2004. No semestre seguinte à graduação entrou para o Programa de Pós-graduação em Informática na mesma universidade.

Ficha Catalográfica

Wagner, Gustavo Nunes

Visualização interativa de modelos massivos de engenharia na indústria de petróleo com o algoritmo de voxels distantes / Gustavo Nunes Wagner ; orientador: Marcelo Gattass ; co-orientador: Alberto Raposo. - 2007.

88f. : il. ; $30 \mathrm{~cm}$

Dissertação (Mestrado em Informática)-Pontifícia Universidade Católica do Rio de Janeiro, Rio de Janeiro, 2006.

Inclui referências bibliográficas.

1. Informática - Teses. 2. Computação Gráfica. 3. Visualização. 4. Modelos Massivos. 5. Oclusão. 6. LOD Hierárquico. 7. Impostores. I. Gattass, Marcelo. II. Raposo, Alberto. III. Pontifícia Universidade Católica do Rio de Janeiro. Departamento de Informática. IV. Título. 


\section{Agradecimentos}

À minha família.

Aos meus orientadores Marcelo Gattass e Alberto Raposo, por todas as idéias e conselhos que foram indispensáveis na elaboração dessa dissertação.

Ao Tecgraf, por ser uma ótima fonte de problemas difíceis e interessantes e um lugar único para trabalhar.

À CAPES, pelo auxílio dado ao longo do curso.

À PUC-Rio e aos seus professores que ensinam com o mesmo empenho tanto as matérias mais simples quanto os assuntos mais complexos.

Ao Instituto de Tecnologia ORT, por ter me ensinado sobre linguagens de programação. 


\section{Resumo}

Wagner, Gustavo Nunes; Gattass, Marcelo. Visualização Interativa de Modelos Massivos de Engenharia na Indústria de Petróleo com o Algoritmo de Voxels Distantes. Rio de Janeiro, 2007. 88p. Dissertação de Mestrado - Departamento de Informática, Pontifícia Universidade Católica do Rio de Janeiro.

Projetos recentes de Estruturas Offshore criaram a necessidade de prototipação virtual de modelos de CAD massivos. Esses modelos tipicamente têm centenas de milhões de triângulos e, por essa razão, não podem ser enviados diretamente para as placas gráficas atuais que podem renderizar interativamente apenas alguns milhões de triângulos. Existem várias abordagens para lidar com esse problema incluindo uma nova estratégia de uso de impostores baseada na visualização de Voxels. Essa estratégia é promissora, já que lida bem com níveis de detalhe, oclusão e armazenamento em memória secundária. Esta dissertação apresenta uma variação do algoritmo de Voxels Distantes (Far Voxels), que é implementada e testada sobre modelos de CAD típicos. Finalmente, a partir desses testes, a dissertação apresenta algumas conclusões e sugestões para trabalhos futuros.

\section{Palavras-chave}

Computação Gráfica; Visualização; Modelos Massivos; Oclusão; LOD Hierarquico; Impostores 


\section{Abstract}

Wagner, Gustavo Nunes; Gattass, Marcelo. Interactive Visualization of Massive Engineering Models in the Oil \& Gas Industry using the Far Voxels Algorithm. Rio de Janeiro, 2007. 88p. MSc. Dissertation Departamento de Informática, Pontifícia Universidade Católica do Rio de Janeiro.

Current projects of Offshore Structures require virtual prototyping of huge CAD models. These models usually have hundreds of millions of triangles and for this reason they cannot be sent directly to current graphical boards that can render interactively only a few millions of triangles. There are many different approaches to deal with this problem including a new impostor strategy based on Voxel visualization. This strategy is promising because it deals well with level of detail, occlusion and out of core model storage. This dissertation presents a variant of the Far Voxels algorithm. This variant is implemented and tested against typical CAD models. Finally, from these tests, the dissertation presents some conclusions and suggestions for future work.

\section{Keywords}

Computer Graphics; Visualization; Massive Models; Occlusion; Hierarchical LODs; Impostors 


\section{Sumário}

1 Introdução

2 Técnicas e Trabalhos Relacionados

3 O Algoritmo de Voxels Distantes

3.1 Níveis de detalhe e hierarquia (HLOD) 25

3.2. Usando voxels para representar modelos 27

3.3. Pré-processamento 33

3.4. Visualização $\quad 42$

$\begin{array}{ll}\text { 3.5. Voxels com filtro de anti-serrilhamento } & 48\end{array}$

3.6. Limitações

4 Implementação $\quad 56$

$\begin{array}{ll}5 \text { Resultados } & 60\end{array}$

$\begin{array}{ll}\text { 5.1 Modelos usados nos testes } & 60\end{array}$

5.2 Testes de desempenho do algoritmo 62

5.2.1 Testes de desempenho com o modelo da P-38 65

5.2.2 Testes de desempenho com o modelo da P-40 69

5.2.3 Testes de desempenho com o modelo da P-50 74

5.3 Testes de desempenho para os voxels com filtro de anti$\begin{array}{ll}\text { serrilhamento } & 78\end{array}$

5.4 Testes de desempenho com cópias dos modelos 80 
6 Conclusões e trabalhos futuros

Bibliografia

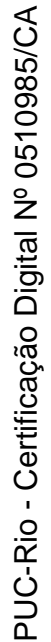




\section{Lista de Figuras}

Figura 2.1 - Exemplos de modelos estudados em algoritmos 18 clássicos de níveis de detalhe.

Figura 2.2 - Exemplo de uma técnica tradicional de LOD usada 20 em modelos massivos.

Figura 2.3 - O mesmo modelo da Figura 2.2 simplificado usando 20 HLODs.

Figura 2.4 - Renderização de um modelo escaneado usando uma 21 hierarquia de pontos.

Figura 2.5 - Representando um Boeing 777 (350 milhões de 22 triângulos) usando a técnica de Voxels Distantes.

Figura 2.6 - Modelo de um navio com 77 milhões de triângulos 23 renderizado com a técnica de R-LOD.

Figura 3.1 - Representação gráfica da hierarquia de níveis de 26 detalhes de um modelo.

Figura 3.2 - Caixa envolvente e representação de voxel.

Figura 3.3 - Diferentes níveis de detalhe para representar o 29 modelo do Teapot.

Figura 3.4 - Diferentes níveis de detalhe para representar a 30 plataforma P-50.

Figura 3.5 - Exemplos de voxels com atributos que variam com a 32 direção de visualização.

Figura 3.6 - Planos de corte de uma octree.

Figura 3.7 - Face cortada pelos planos de corte da octree. 
Figura 3.8 - Limite de que determina quando as faces deverão ser duplicadas.

Figura 3.9 - Voxels gerados com cores erradas devido a um 37 tratamento incorreto das superfícies ocultas.

Figura 3.10 - Exemplo de configuração de um traçado de raios 39 típico.

Figura 3.11 - Falhas visuais geradas por objetos muito finos 49 sendo representados por voxels.

Figura 3.12 - As mesmas falhas da figura 3.11, representadas 49 sem ampliação.

Figura 3.13 - Filtro de anti-serrilhamento no modelo SKID_ABC.

Figura 3.14 - Filtro de anti-serrilhamento no modelo P-50.

51

Figura 3.15 - Voxels atravessados por muitos raios indicando a 52 existência de objetos que ocupam apenas uma pequena parte do volume do voxel.

Figura 3.16 - Casos comuns de voxels transparentes encontrados 53 durante o traçado de raios.

Figura 3.17 - Visualização envolvendo várias plataformas que 55 podem ser movimentadas individualmente.

Figura 5.1 - Distribuição de nós na P-38.

Figura 5.2 - Distribuição de nós na P-40.

Figura 5.3 - Distribuição de nós na P-50.

62

Figura 5.4 - Caminho percorrido durante o teste de desempenho 65 na $\mathrm{P}-38$.

Figura 5.5 - Imagens de cada trecho do caminho percorrido 66 durante os testes na P-38. 
Figura 5.6 - Resultado em quadros por segundo dos testes de desempenho na P-38.

Figura 5.7 - Primeiro gráfico com resultados da análise realizada para localizar o gargalo na navegação da P-38.

Figura 5.8 - Segundo gráfico com resultados da análise realizada 68 para localizar o gargalo na navegação da P-38.

Figura 5.9 - Caminho percorrido durante o teste de desempenho 70 na P-40.

Figura 5.10 - Imagens de cada trecho do caminho percorrido 71 durante os testes na P-40.

Figura 5.11 - Resultado em quadros por segundo dos testes de 71 desempenho na P-40.

Figura 5.12 - Primeiro gráfico com resultados da análise realizada 72 para localizar o gargalo na navegação da P-40.

Figura 5.13 - Segundo gráfico com resultados da análise 73 realizada para localizar o gargalo na navegação da P-40.

Figura 5.14 - Caminho percorrido durante o teste de desempenho 74 na P-50.

Figura 5.15 - Imagens de cada trecho do caminho percorrido 75 durante os testes na P-50.

Figura 5.16 - Resultado em quadros por segundo dos testes de 76 desempenho na P-50.

Figura 5.17 - Primeiro gráfico com resultados da análise realizada 77 para localizar o gargalo na navegação da P-50.

Figura 5.18 - Segundo gráfico com resultados da análise 77 realizada para localizar o gargalo na navegação da P-50. 
Figura 5.19 - Gráfico comparativo do desempenho usando o filtro anti-serrilhamento no modelo da P-38.

Figura 5.20 - Gráfico comparativo do desempenho o filtro anti- 79 serrilhamento no modelo da P-40.

Figura 5.21 - Gráfico comparativo do desempenho usando o filtro 80 anti-serrilhamento no modelo da P-50.

Figura 5.22 - 25 Cópias da P-50 posicionados lado-a-lado.

Figura 5.23 - Desempenho obtido com as 25 cópias da P-50.

Figura 5.24 - 9 Cópias de cada um dos 3 modelos de teste 82 posicionados lado-a-lado, totalizando 27 modelos.

Figura 5.25 - Desempenho obtido com os 27 modelos. 


\section{Lista de Tabelas}

Tabela 5.1 - Características dos modelos usados nos testes. 